Check for updates

Cite this: RSC Adv., 2018, 8, 2426

Received 30th November 2017 Accepted 28th December 2017

DOI: 10.1039/c7ra12931a

rsc.li/rsc-advances

\section{Adsorption of toluene with water on zeolitic imidazolate framework-8/graphene oxide hybrid nanocomposites in a humid atmosphere}

\author{
Fuchen Chu, (D) Yue Zheng, Boyuan Wen, Lin Zhou, Jun Yan and Yunlin Chen (DD*
}

\section{Introduction}

With the rapid development of industry, the release of volatile organic compounds (VOCs) into the atmosphere has become a global threat to the ecological environment. As a principle member of VOCs, toluene is widely used in various industrial processes and has created severe air pollution, especially in some developing countries. Many techniques have been used for abating the emission of toluene and other VOCs, including condensation, ${ }^{1}$ adsorption, ${ }^{2,3}$ catalytic oxidation, ${ }^{4}$ and biodegradation treatments. ${ }^{5,6}$ The most preferable option for removing VOCs from polluted air is adsorption. Adsorbents play a vital role in enhancing VOC uptake capacity. Up to now, many materials have been investigated to find a more cost-effective adsorbent to deal with VOCs. Although these traditional absorbents were widely used in the past, their disadvantages of being invalid in complex environments and having a low capacity have limited their wider use in industrial application. ${ }^{7}$

In recent years, zeolitic imidazolate frameworks (ZIFs), the sub-family of metal-organic frameworks (MOFs), have been rapidly developed. ZIFs structures are constructed from tetrahedral metal ions (M) connected by imidazolate (Im) linkers. By this linking procedure, a framework is built up, whose structures are similar to those of zeolites having tetrahedral unit. ${ }^{8}$ They are considered to be a promising alternative for VOCs

Institute of Applied Micro-Nano Materials, School of Science, Beijing Jiaotong University, Beijing 100044, People's Republic of China. E-mail: ylchen@bjtu.edu.cn; Tel: +861051688483 adsorption due to their unique properties, such as ultrahigh surface areas, tunability and chemical stabilities. ${ }^{8,9}$ ZIFs have attracted a growing research interest in their potential applications in gas separation, ${ }^{10,11}$ catalysis, ${ }^{12}$ adsorption ${ }^{13,14}$ and drug delivery. ${ }^{15}$

On the other hand, the graphene and graphene-based materials have attracted considerable attention owing to its facile synthesis and excellent properties. Graphene oxide (GO), a typical sheet-shaped material, has been widely utilized in decorating composite materials with promising adsorptive properties. Actually, many studies recently showed the MOFsbased graphite oxide composites for different applications. Furthermore, these studies have confirmed that the incorporation of GO could improve the adsorption capacity of MOFs. For instance, Yongde Xia and co-workers reported a simple and in situ synthesis method to prepare ZIF-8/GO composites in aqueous solution, which showed enhanced $\mathrm{CO}_{2}$ adsorption energy and significant $\mathrm{CO}_{2}$ uptake capacity. ${ }^{16}$ Recently, Dong et al. reported high-performance mixed matrix membranes for efficient separation of $\mathrm{CO}_{2} / \mathrm{N}_{2}$ by taking advantage of novel ZIF8/GO composite filler. ${ }^{17}$ Zhou and colleagues found that the composites of ZIF-8/GO composites exhibited enhanced VOCs adsorption capacity. ${ }^{18}$ However, the research on VOCs adsorption performance of ZIFs/GO composites in aqueous system is very limited so far, as a majority of adsorption experiments of MOF/GO composites are under ideal conditions without considering the effects of water. ${ }^{17-19}$

As is known to all, water vapor is omnipresent. It often presents in polluted air and industrial gas streams, which may 
decrease the VOCs adsorption because of strong competitive adsorption. ZIF-8 is expected to allow VOCs molecules entering in it more easily than water molecules due to its hydrophobic inner channels, which has been confirmed in previous researches. ${ }^{\mathbf{2 0 2 1}}$ However, GO is extremely hydrophilic and water molecules can be adsorbed with high affinity, ${ }^{22,23}$ which may lead to a decrease in the amount of adsorption. Little attention has been focused on the study of VOCs adsorption on ZIF-8/GO composites in humid atmosphere so far. Therefore, it is significant to investigate the competitive adsorption of the ZIF8/GO for VOCs/water. In this work, the ZIF-8/GO composites with different proportions of GO were synthesized and characterized. The breakthrough curves of toluene and water mixture were measured at different relative humidity by fixed bed experiments for the first time. The reusability of ZIF-8/GO was also explored, and ZIF-8/GO composites were demonstrated as potential adsorbents for the removal of toluene in humid environment.

\section{Experimental}

\subsection{Materials}

Zinc nitrate hexahydrate $\left(\mathrm{Zn}\left(\mathrm{NO}_{3}\right)_{2} \cdot 6 \mathrm{H}_{2} \mathrm{O}, 98 \%\right)$ was obtained from Acros. Methanol ( $\mathrm{MeOH}, 98 \%)$ and 2-methyl imidazole (Hmim, 99\%) were purchased from Sigma-Aldrich. All chemicals were of analytical grade and were used as received from vendors without any further purification.

\subsection{Materials synthesis}

2.2.1. Synthesis of ZIF-8. ZIF-8 was synthesized according to the literature procedure reported by Wiebcke et al. ${ }^{24}$ In brief, $0.734 \mathrm{~g}$ of $\mathrm{Zn}\left(\mathrm{NO}_{3}\right)_{2} \cdot 6 \mathrm{H}_{2} \mathrm{O}$ and $0.811 \mathrm{~g}$ of 2-methyl imidazole were dissolved in $50 \mathrm{~mL}$ of methanol separately. The zinc nitrate solution then added to the 2-MeIM solution, and the mixture was stirred for $1 \mathrm{~h}$ at room temperature. The metal: ligand : MeOH molar ratios of the mixture were $1: 4: 1250$. After that, the solution was centrifuged and the precipitated crystals were washed with methanol for three times. Finally, the product was dried at $70{ }^{\circ} \mathrm{C}$ overnight and the amount of imidazole in the final construct was at a ratio of $71 \%$ by weight.

2.2.2. Synthesis of ZIF-8/GO hybrid composites. ZIF-8/GO hybrid composites were synthesized according to the literature procedure reported by Zhou et al. ${ }^{18}$ Firstly, calculated amount of GO powder was dispersed in methanol and sonicated for $6 \mathrm{~h}$. Next, GO after ultrasonic treatment was added during the synthesis of ZIF-8 in the stirring process. Finally, the mixture was centrifuged and washed for 3 times with methanol and dried at $60{ }^{\circ} \mathrm{C}$ for $6 \mathrm{~h}$. All the synthesized materials used in adsorption studies were powders. The resulting composites with variable GO content were denoted as ZIF-8/GO- $X$ which indicated $X$ wt $\%$ of GO. The ZIF-8/GO- $X$ samples were synthesized and designated as ZG- $X$ (ZG-2, ZG-4, ZG-7, ZG-10, ZG-15, ZG-20, ZG-25) according to the weight percentage of GO.

\subsection{Characterization}

X-ray diffraction (XRD) patterns were carried out on a BRUKER D8 ADVANCE diffractometer under the condition of $\mathrm{Cu} \mathrm{K} \alpha$ radiation at $36 \mathrm{kV}$ and $20 \mathrm{~mA}$. The scope of the scan was from $3^{\circ}$ to $60^{\circ}$ with a step size of $0.01^{\circ}$. Fourier transform infrared spectroscopy (FTIR) was obtained on a SPECTRUM 400 spectrometer with scan range of $2000-500 \mathrm{~cm}^{-1}$. The scanning electron microscope (SEM) images of the samples were taken on a FEI Quanta 650 field emission scanning electronic microscope at an accelerating voltage of $10 \mathrm{kV}$. Thermogravimetric analysis (TGA) was performed on a TA-SDT Q600 instrument in the temperature range from room temperature to $700{ }^{\circ} \mathrm{C}$ with a heating rate of $10{ }^{\circ} \mathrm{C} \min ^{-1}$ in nitrogen atmosphere. Nitrogen adsorption isotherms were measured at $77 \mathrm{~K}$ using JWBK122W. Before the analysis, all samples were degassed at $160{ }^{\circ} \mathrm{C}$ for $18 \mathrm{~h}$ under vacuum. The specific surface area was determined with the Brunauer-Emmett-Teller (BET) method based on the data from $\mathrm{N}_{2}$ adsorption within the range of relative pressure from 0.005 to 0.3 .

\subsection{Adsorption experiments}

Adsorption experiments were used to assess the adsorption properties of toluene on synthesized materials and the effect of

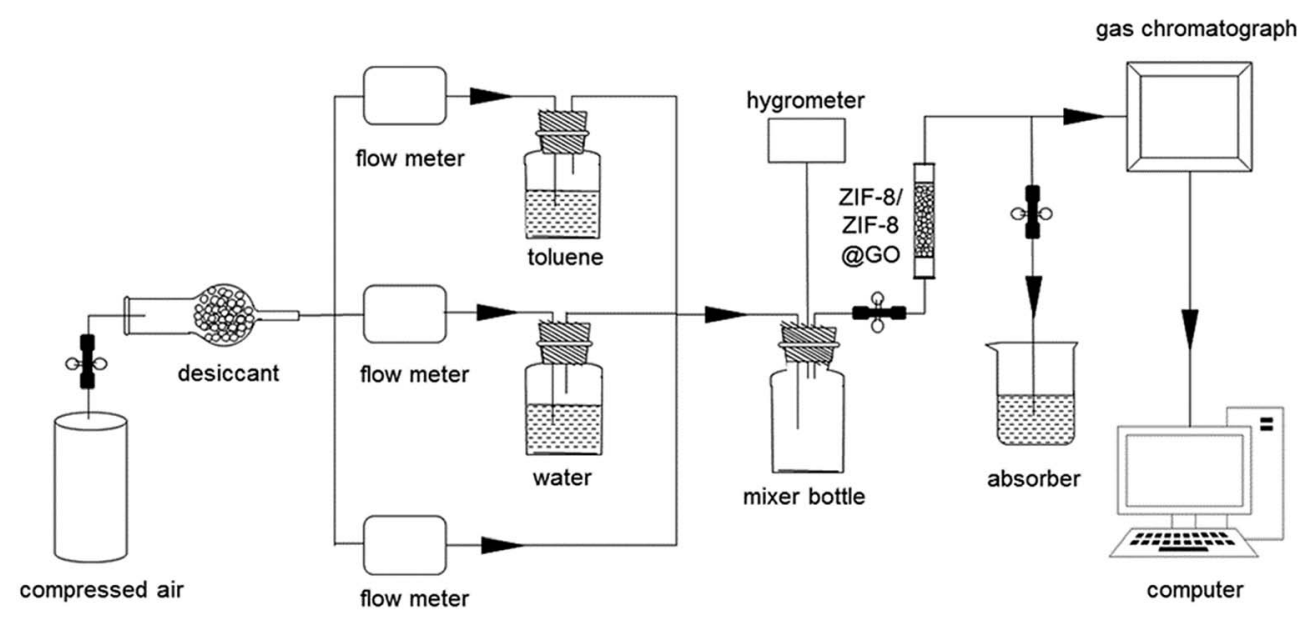

Fig. 1 Schematic diagram of the adsorption experimental system. 
different humidity on adsorption. The experimental setup is shown in Fig. 1 and all adsorbents were evacuated at $120^{\circ} \mathrm{C}$ for $6 \mathrm{~h}$ under vacuum prior to the experiments. The total flow-rate

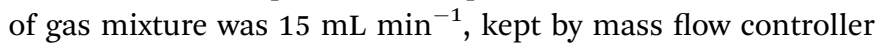
(D08-3F, Sevenstar Co. Ltd., China). After that, the gas flow of pure air was divided into three branches to blow out a gas mixture of toluene and water with a certain concentration. The humidity of the gas could be changed by adjusting the flow rate of dry air with an accuracy of $\pm 2.0 \%$. Finally, the gas mixture was thoroughly mixed in the buffer bottle and then delivered to the fixed-bed. The fixed-bed was made of a quartz tube with $5.0 \mathrm{~mm}$ inner diameter and $10.0 \mathrm{~cm}$ in height. The collected gas from the inlet and outlet of the tube was continuously detected by using a gas chromatography (GC). After adsorption was finished, the adsorbent was washed by methanol and the obtained solid was dried at $120{ }^{\circ} \mathrm{C}$ for $6 \mathrm{~h}$ under vacuum for next adsorption run.

\section{Results and discussion}

\subsection{Characterization of ZIF-8 crystals and ZG- $X$ composites}

The X-ray diffraction patterns taken from GO, ZIF-8 and ZIF-8/ GO with different GO contents were shown in Fig. 2. The XRD results of the ZIF-8/GO composites also exhibited the reflection of pure ZIF-8 crystalline, indicating the presence of GO had no effect on the formation of the ZIF-8 during the synthesis procedure, even if the content of GO was up to $25 \mathrm{wt} \%$. However, the intensity of the characteristic peaks of ZIF-8 was waning with an increase of GO content, which would be attributed to a quite strong binding of GO to ZIF-8. All the results suggested that GO sheets may form strong interactions with ZIF-8 crystals as an integral part of ZIF-8/GO.

Fig. 3 showed the FTIR spectra of GO, ZIF-8 and ZG- $X$ composites. As we can see in Fig. 3, all the synthesized ZG- $X$

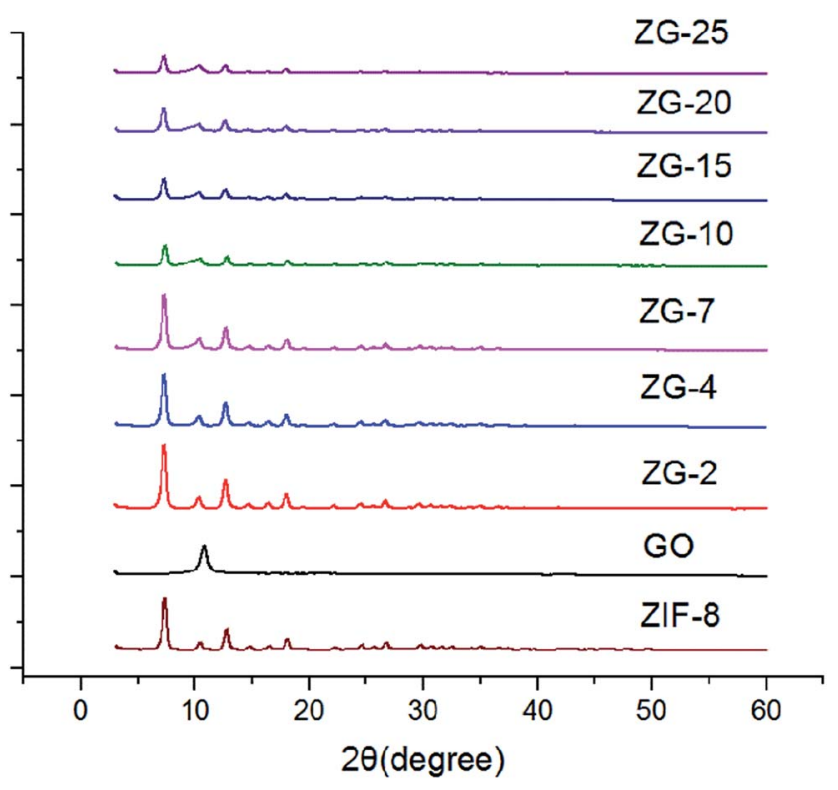

Fig. 2 XRD patterns of GO, ZIF-8 and ZG-X composites.

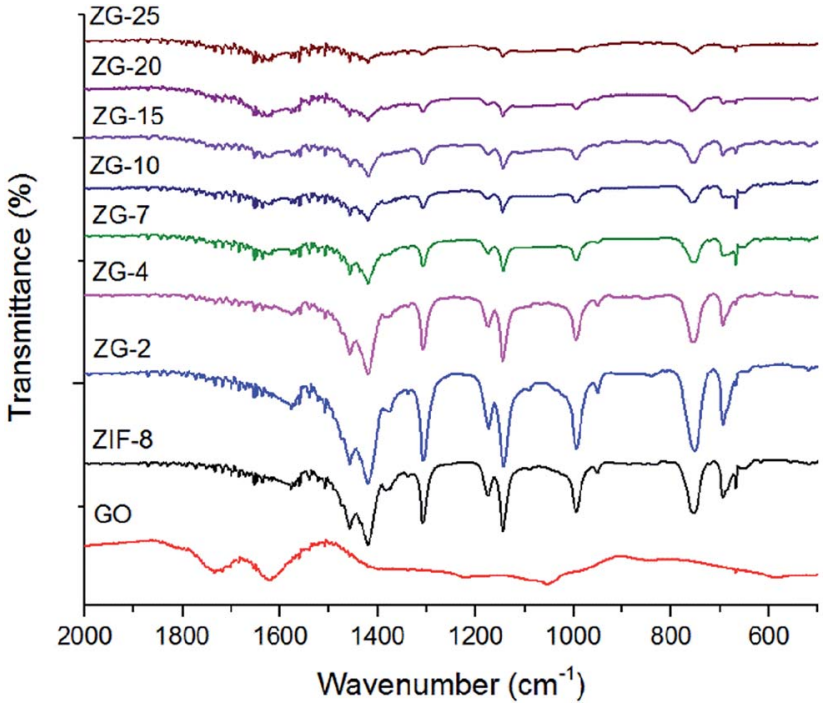

Fig. 3 FTIR spectra of GO, ZIF-8 and ZG-X composites.

composites showed the similar FTIR spectra with ZIF-8. The primary part of the absorption bands for ZIF-8 and ZG- $X$, for instance, the bands at $1584 \mathrm{~cm}^{-1}$ were due to the $\mathrm{C}=\mathrm{N}$ stretch mode and the bands at 1150 and $995 \mathrm{~cm}^{-1}$ were assigned as the $\mathrm{C}-\mathrm{N}$ stretch mode, were connected to the vibrations of the imidazole units. Besides, the peaks at $1250-1500 \mathrm{~cm}^{-1}$ were associated with the imidazole ring stretching and the peaks below $1250 \mathrm{~cm}^{-1}$ were correspond to the out-of-plane bending and in-plane bending of the imidazole ring. Moreover, the typical absorption bands for GO were the $\mathrm{C}-\mathrm{O}$ vibrations at $1068 \mathrm{~cm}^{-1}$ and the $\mathrm{C}=\mathrm{O}$ stretching vibrations at $1790 \mathrm{~cm}^{-1}$ and there were no such peaks or bands existing in the ZG- $X$ composites. The FTIR results clearly confirmed that GO had successfully been loaded onto ZIF-8 and the strong interactions were formed between ZIF-8 and GO during impregnation.

Thermogravimetric analysis (TGA) of ZIF-8 and ZIF-8/GO composites was presented in Fig. 4 and the result curves

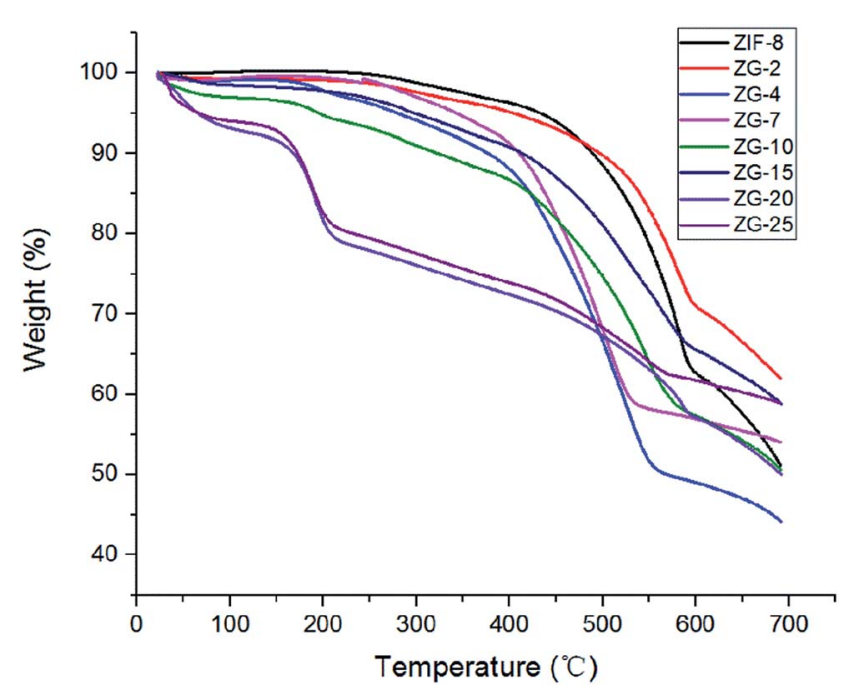

Fig. 4 TGA curves of ZIF-8 and ZG- $X$ composites. 
showed high thermal stability. The TGA curve for the sample ZIF-8 showed a little weight loss before $350{ }^{\circ} \mathrm{C}$, which may be attributed to the removal of residual solvent or some guest molecules. $^{25}$ In contrast, a sharp weight loss was observed between $400{ }^{\circ} \mathrm{C}$ and $600{ }^{\circ} \mathrm{C}$, corresponding to the decomposition of organic imidazolate species and the collapse of the ZIF-8 structure. ${ }^{25}$ Compared with ZIF-8, the TGA results of the ZIF-8/ GO composites exhibited slightly increased weight losses at approximately the same temperature, indicating a strong interaction between GO sheets and ZIF-8. However, a rapid decomposition of ZG-20 and ZG-25 started at $200{ }^{\circ} \mathrm{C}$, which was ascribed to the removal of the excessive amount of GO that cannot be strongly combined. The TGA results showed that the ZIF-8 and ZG- $X$ composites with low content of GO were thermally stable, which made them adaptive for industrial applications.

As shown in Fig. 5, the morphologies and sizes of ZIF-8 and ZIF-8/GO composites were characterized using scanning electron microscopy (SEM). The pristine ZIF-8 showed uniform hexagonal morphology with the particle sizes in the range of 50$100 \mathrm{~nm}$, as shown in Fig. 5(a). Furthermore, similar hexagonal morphology and particle size were also exhibited on the ZG- $X$ composites, with the ZIF-8 particles being stabilized within the GO sheets, as shown in Fig. 5(b-h). As can be seen from the Fig. 5(e-h), the GO sheets showed wrinkles and folding on the edges and the ZIF-8 particles grew in situ on the surface of sheets with increasing content of GO. It can be inferred that the dense GO sheets could be performed as a platform for the nanoscale ZIF-8 crystals growth.

The textural properties of the ZIF-8 and ZG- $X$ composites could be obtained from $\mathrm{N}_{2}$ adsorption isotherms at $77 \mathrm{~K}$, as shown in Fig. 6. It can be noticed that the curve displayed a steep rise at low relative pressures owing to the presence of microporosity in these porous materials and another rise observed at high relative pressures. In general, all the samples displayed type I isotherm profiles, implying the microporous
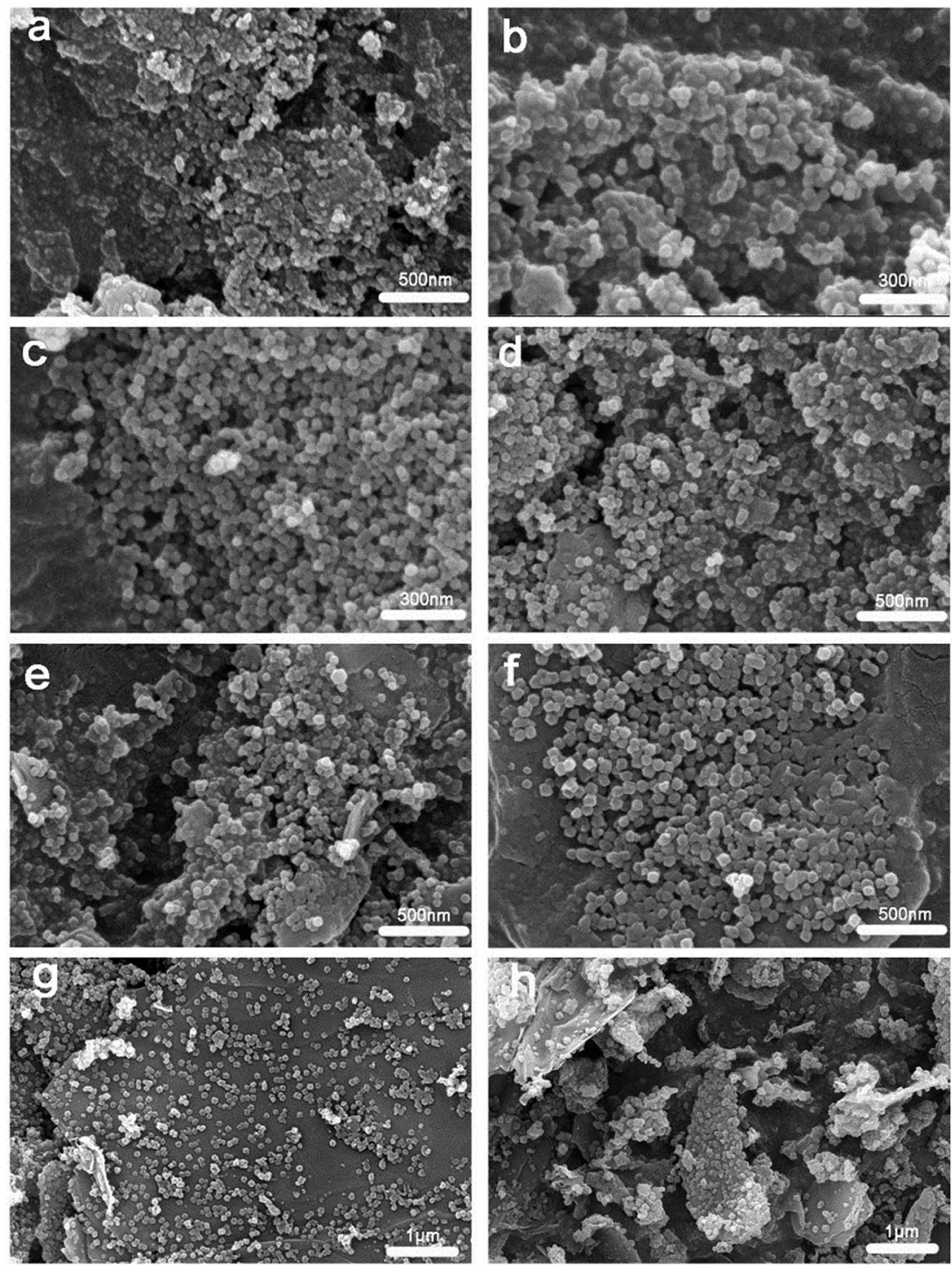

Fig. 5 Representative SEM images of as-synthesized ZIF-8 sample and ZG-X composites: (a) ZIF-8, (b) ZG-2, (c) ZG-4, (d) ZG-7, (e) ZG-10, (f) ZG15, (g) ZG-20, (h) ZG-25. 


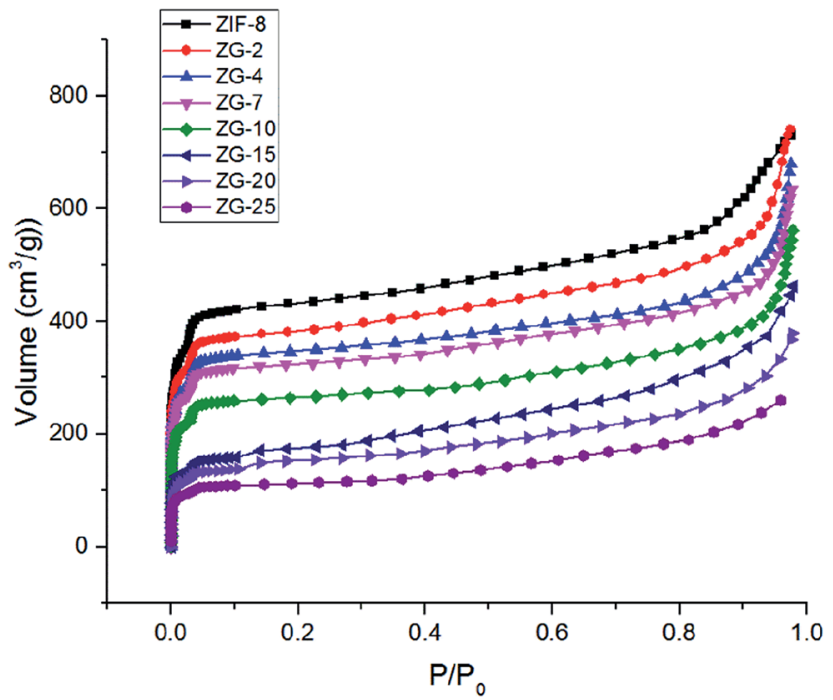

Fig. 6 Nitrogen adsorption isotherms measured at $77 \mathrm{~K}$ for ZIF- 8 and ZG-X composites.

structure were in dominant. However, the small slope observed at medium relative pressures might due to the existence of mesoporosity. The surface areas of the ZG- $X$ composites could be modulated by adjusting the GO content in the composites as summarized in Table 1. The BET surface area of ZIF-8 was 1388 $\mathrm{m}^{2} \mathrm{~g}^{-1}$ and the Langmuir surface area was $1954 \mathrm{~m}^{2} \mathrm{~g}^{-1}$. It was found that the surface areas of $\mathrm{ZG}-X$ composites decreased with increasing GO content, as showed in Table 1 . These results demonstrated that the nonporous GO may block the pore channels of ZIF-8 and cause a decrease in surface areas of the composites.

\subsection{Adsorption studies}

3.2.1. Adsorption performances of ZIF-8 and ZG- $X$ for toluene in humidity. Fig. 7 showed the breakthrough curves of toluene obtained at $303 \mathrm{~K}$ and $55 \%$ relative humidity for all synthetic adsorbents. The breakthrough times and working capacities of toluene were recorded in Table 2 under conditions of similar inlet concentration. In Table 2 , breakthrough time $\left(t_{\mathrm{b}}\right)$ stands for time when the outlet concentration is $2 \%$ of the inlet concentration and the time when the outlet concentration is close to that of inlet is represented by equilibrium time $\left(t_{\mathrm{e}}\right)$. The equilibrium adsorption capacity $\left(Q_{\mathrm{e}}\right)$ was calculated as follows:

$$
Q_{\mathrm{e}}=\frac{10^{-6} F C_{0}}{M}\left(t_{\mathrm{e}}-\int_{0}^{t_{\mathrm{e}}} \frac{C_{\mathrm{i}}}{C_{0}} \mathrm{~d} t\right)
$$

where $F\left(\mathrm{~mL} \mathrm{~min}^{-1}\right)$ is the gas flow rate and $M(\mathrm{~g})$ represents the amount of adsorbent; $C_{\mathrm{i}}\left(\mathrm{mg} \mathrm{m}^{-3}\right)$ and $C_{0}\left(\mathrm{mg} \mathrm{m}^{-3}\right)$ are the

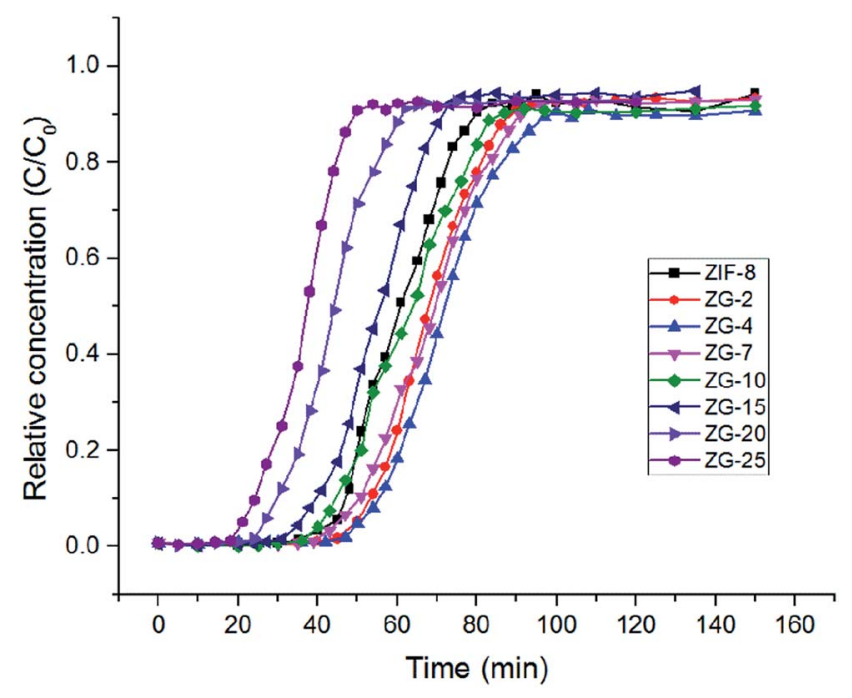

Fig. 7 Breakthrough curves of adsorption of toluene by ZIF- 8 and ZG$X$ composites at $303 \mathrm{~K}$ and $55 \%$ relative humidity.

outlet concentration and inlet concentration of toluene, respectively.

According to the experimental results, it can be seen that the breakthrough time of the ZG- $X$ composites with low content of GO became longer which means a better adsorbing performance. Surprisingly, when the content of GO reached $4 \mathrm{wt} \%$, the breakthrough time of the $\mathrm{ZG}-X$ composites decreased with an increased amount of GO. From these breakthrough data, it was calculated that ZIF-8, ZG-2 and ZG-4 took up toluene adsorption at 97, 109 and $116 \mathrm{mg} \mathrm{g}^{-1}$, respectively. It is generally believed that VOCs adsorption capacities of porous materials depend on large specific area. However, although samples such as ZG-2 and ZG-4 possess a lower surface area, these samples exhibited higher adsorption capacity. The synthetic ZG- $X$ composites showed good toluene adsorption capacity with the content of GO below $7 \mathrm{wt} \%$ and this clearly indicated that the introduction of GO could still increase the affinity of ZIF-8 toward toluene in water vapor. It is worth noting that compared with the pristine ZIF-8, sample ZG-4 had the highest toluene adsorption capacity of $116 \mathrm{mg} \mathrm{g}^{-1}$, increased by $19 \%$. The result of this part was consistent with the early report. ${ }^{18}$ This unusual uptake was not closely associated with specific surface area and could be attributed to the synergistic effect of ZIF-8 and GO. $\pi-\pi$ interaction between GO layers and toluene might be a suitable mechanism, since both the two species have benzene rings. ${ }^{26}$ Moreover, the formation of hydrogen bonds between the hydroxyl groups on GO and imidazolate unit that is part of ZIF-8 might increase the interaction degree between GO layers and ZIF-8 crystals. ${ }^{16}$ As a result, new pores were formed

Table 1 Surface areas of the as-synthesized ZIF- 8 samples and ZG-X composites

\begin{tabular}{lccccccc}
\hline Sample & ZIF-8 & ZG-2 & ZG-4 & ZG-7 & ZG-10 & ZG-15 & ZG-20 \\
\hline BET surface area $\left(\mathrm{m}^{2} \mathrm{~g}^{-1}\right)$ & 1388 & 1237 & 1112 & 1038 & 845 & 578 & 506 \\
Langmuir surface area $\left(\mathrm{m}^{2} \mathrm{~g}^{-1}\right)$ & 1954 & 1731 & 1567 & 1454 & 1192 & 832 & 731
\end{tabular}


Table 2 Column characteristics from breakthrough curves in terms of materials under the same humidity

\begin{tabular}{llllll}
\hline Sample & RH $(\%)$ & $t_{\mathrm{b}}(\mathrm{min})$ & $t_{\mathrm{e}}(\mathrm{min})$ & $Q_{\mathrm{e}}\left(\mathrm{mg} \mathrm{g}^{-1}\right)$ & $C / C_{0}$ \\
\hline ZIF-8 & 55 & 35 & 81 & 97 & 0.91 \\
ZG-2 & 55 & 40 & 89 & 109 & 0.91 \\
ZG-4 & 55 & 43 & 97 & 116 & 0.89 \\
ZG-7 & 55 & 39 & 92 & 109 & 0.92 \\
ZG-10 & 55 & 36 & 84 & 100 & 0.90 \\
ZG-15 & 55 & 31 & 74 & 88 & 0.93 \\
ZG-20 & 55 & 24 & 62 & 71 & 0.92 \\
ZG-25 & 55 & 18 & 51 & 59 & 0.91
\end{tabular}

between the interface of ZIF-8 and GO structures and the adsorption capacities of ZIF-8/GO composites could be enhanced. Therefore, the addition of GO into the composites can still affect the textural properties and influence remarkably on the toluene uptake capacities in high humidity conditions. Moreover, the composites with higher GO content (more than 4 wt\%) showed decreased adsorption capacity, which is different with previous studies. ${ }^{16,18}$ This phenomenon may be related to the hydrophilicity of GO and would be discussed in the following study.

3.2.2. Influence of humidity on adsorption. In order to explore the effect of water vapor on adsorption, breakthrough curves of toluene through a fixed bed packed with ZG-4 were tested at different relative humidity (Fig. 8). All the other experimental conditions and methods of calculation were consistent with the above experiment (3.2.1). The breakthrough times and adsorption capacities at relative humidity of $10 \%$, $55 \%, 80 \%$ were listed in Table 3 . It was obvious that the vapor had a negative influence on the breakthrough curves, implying a gradually adsorption capacity decreasing due to presence of water vapor in the feed stream. The adsorption capacities of toluene on ZG-4 were decreased from $123 \mathrm{mg} \mathrm{g}^{-1}$ at $10 \mathrm{RH} \%$ to 116 and $110 \mathrm{mg} \mathrm{g}^{-1}$ at 55 and $80 \mathrm{RH} \%$, respectively. The

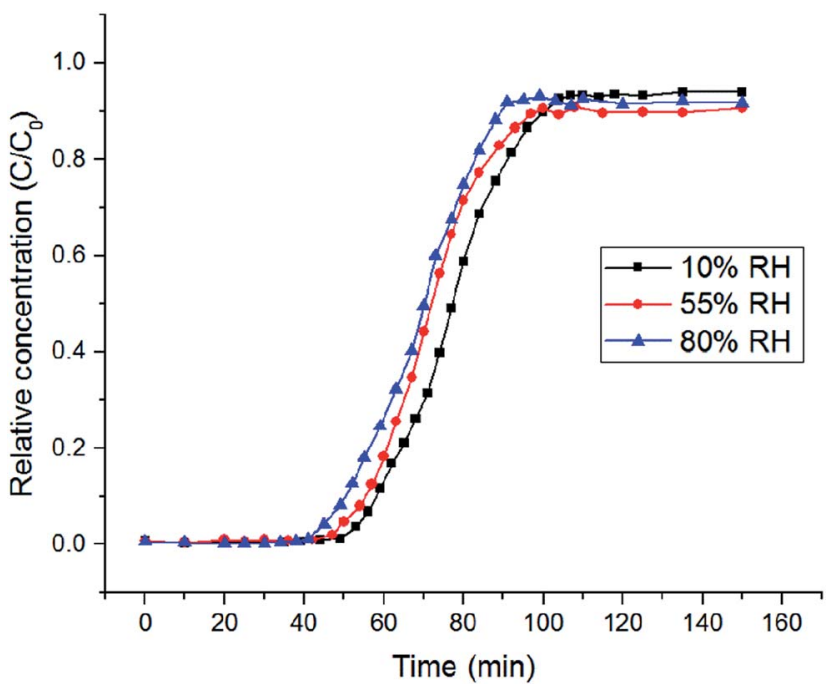

Fig. 8 Breakthrough curves of adsorption of toluene by ZG-4 at $308 \mathrm{~K}$ in different humidity.
Table 3 Column characteristics from breakthrough curves in terms of ZG-4 at different humidity

\begin{tabular}{lllll}
\hline $\mathrm{RH}(\%)$ & $t_{\mathrm{b}}(\mathrm{min})$ & $t_{\mathrm{e}}(\mathrm{min})$ & $Q_{\mathrm{e}}\left(\mathrm{mg} \mathrm{g}^{-1}\right)$ & $C / C_{0}$ \\
\hline 10 & 47 & 104 & 123 & 0.93 \\
55 & 43 & 97 & 116 & 0.89 \\
80 & 41 & 91 & 110 & 0.92
\end{tabular}

decreasing amount of adsorbed toluene could be attributed to the competitive adsorption of water molecules on the surfaces of GO because of its hydrophilicity. As a result, a part of the pores of ZG-4 composite would be occupied and fewer sites were accessible for toluene adsorption. By calculation, approximately $11.8 \%$ of the adsorbed amount of toluene was suppressed when the relative humidity reached $80 \%$. However, compared with the enhancement of adsorption properties caused by the addition of GO, the inhibitory effect on the amount of adsorption with RH was insignificant when the content of GO was low. Thus, the combination of ZIF-8 and GO is still an effective method to improve the adsorption capacity of toluene at relatively high RH conditions. To the best of our knowledge, this is the first report on the effect of water vapor on the adsorption of VOCs by ZIF-8/GO composite materials.

\subsection{Recyclability of the adsorbent}

In commercial applications of adsorptive removal, recyclability of the adsorbent is an important parameter to be considered. Therefore, the regenerated ZG-4 was reused in the subsequent adsorption process for five times under the same conditions to explore the reusability. It can be seen from the results in Fig. 9 that the adsorption of toluene on ZG-4 was slightly decreased after first reuse. However, the adsorbent showed no apparent loss in toluene removal from the second to fifth cycles. Therefore, it suggested that the ZG-4 can be reused for the adsorptive removal of toluene with excellent regeneration and adsorption-

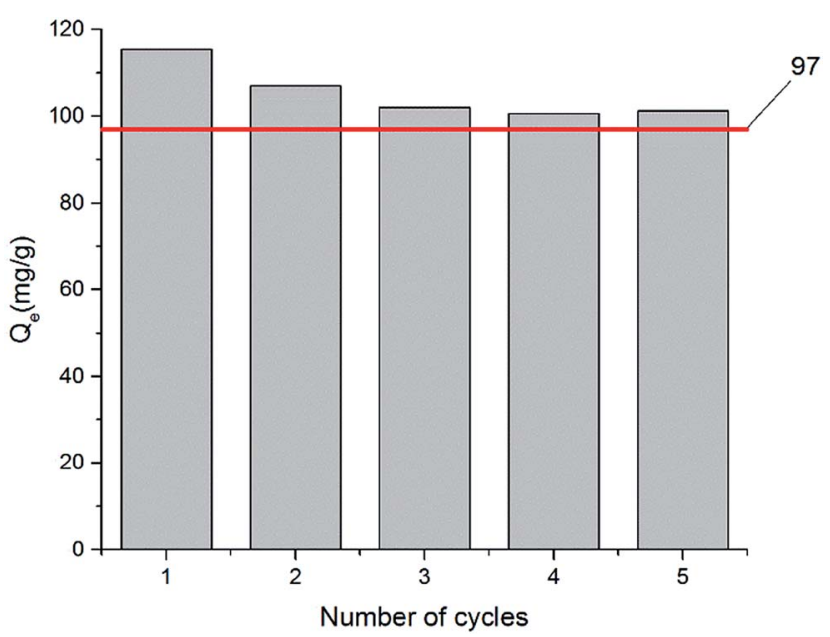

Fig. 9 Recyclability of ZG-4 for adsorption of toluene at 55\% relative humidity. The red line shows the adsorption capacity of toluene over ZIF-8. 
desorption stability. Moreover, the adsorption capacity of regenerated ZG-4 was still superior to the performance of pristine ZIF-8, as demonstrated in Fig. 9.

\section{Conclusion}

The ZIF-8/GO composites with various contents of GO were successfully fabricated, and we found that the textural properties of ZIF-8/GO composites could be tuned by the control over the concentration of GO and GO sheets acted as potential structure-directing agents for the growth of ZIF-8 crystals. The composites prepared in this work were firstly applied for toluene adsorption in different humidity. The experimental results showed that the surface areas of the $\mathrm{ZG}-X$ composites were smaller than those of pristine ZIF-8, but the ZG- $X$ composites exhibited a higher toluene adsorption capacity compared to the pure ZIF-8 as the content of GO below $7 \mathrm{wt} \%$ in humid environment. Adsorption experiments demonstrated that the ZIF-8/GO composites with the GO content of $4 \mathrm{wt} \%$ had the highest toluene adsorption capacity of $116 \mathrm{mg} \mathrm{g}^{-1}$ at relative humidity of $55 \%$, increased by $19 \%$ comparing with pristine ZIF-8. Even at relatively humidity of $80 \%$, the synthesized ZG-4 still exhibited a higher toluene uptake capacity than pristine ZIF-8 crystals. Moreover, the ZG-4 could be efficiently regenerated and reused for adsorption over five recycle steps. These adsorption results revealed that the ZIF-8/GO composites with low content of GO may be used as effective adsorbents to remove VOCs in actual industrial production.

\section{Conflicts of interest}

There are no conflicts to declare.

\section{Acknowledgements}

This work was supported by the National Natural Science Foundation of China No. 21376026.

\section{References}

1 B. Belaissaoui, Y. Le Moullec and E. Favre, Energy, 2016, 95, 291-302.

2 D. Romero, D. Chlala, M. Labaki, S. Royer, J. Bellat, I. Bezverkhyy, J. Giraudon and J. Lamonier, Catalysts, 2015, 5, 1479-1497.

3 S. Xian, Y. Yu, J. Xiao, Z. Zhang, Q. Xia, H. Wang and Z. Li, RSC Adv., 2015, 5, 1827-1834.
4 M. S. Kamal, S. A. Razzak and M. M. Hossain, Atmos. Environ., 2016, 140, 117-134.

5 Z. Cheng, L. Lu, C. Kennes, J. Yu and J. Chen, J. Hazard. Mater., 2016, 303, 83-93.

6 J. Li, M. Li, J. Zhang, D. Ye, X. Zhu and Q. Liao, Biochem. Eng. J., 2013, 75, 39-46.

7 N. Qi, W. S. Appel, M. D. LeVan and J. E. Finn, Ind. Eng. Chem. Res., 2006, 45, 2303-2314.

8 K. S. Park, Z. Ni, A. P. Côté, J. Y. Choi, R. Huang, F. J. Uriberomo, H. K. Chae, M. O'Keeffe and O. M. Yaghi, Proc. Natl. Acad. Sci. U. S. A., 2006, 103, 10186.

9 Y. Pan, Y. Liu, G. Zeng, L. Zhao and Z. Lai, Chem. Commun., 2011, 47, 2071-2073.

10 H. An, S. Park, H. T. Kwon, H. Jeong and J. S. Lee, J. Membr. Sci., 2017, 526, 367-376.

11 H. R. Amedi and M. Aghajani, J. Nat. Gas Sci. Eng., 2016, 35, 695-702.

12 J. Liu, R. Li, Y. Wang, Y. Wang, X. Zhang and C. Fan, J. Alloys Compd., 2017, 693, 543-549.

13 M. Shams, M. H. Dehghani, R. Nabizadeh, A. Mesdaghinia, M. Alimohammadi and A. A. Najafpoor, J. Mol. Liq., 2016, 224, 151-157.

14 S. Li, X. Zhang and Y. Huang, J. Hazard. Mater., 2017, 321, 711-719.

15 Z. Tian, X. Yao and Y. Zhu, Microporous Mesoporous Mater., 2017, 237, 160-167.

16 B. Chen, Y. Zhu and Y. Xia, RSC Adv., 2015, 5, 30464-30471.

17 L. Dong, M. Chen, J. Li, D. Shi, W. Dong, X. Li and Y. Bai, J. Membr. Sci., 2016, 520, 801-811.

18 Y. Zhou, L. Zhou, X. Zhang and Y. Chen, Microporous Mesoporous Mater., 2016, 225, 488-493.

19 X. Zhou, W. Huang, J. Shi, Z. Zhao, Q. Xia, Y. Li, H. Wang and Z. Li, J. Mater. Chem. A, 2014, 2, 4722-4730.

20 K. Zhang, R. P. Lively, C. Zhang, W. J. Koros and R. R. Chance, J. Phys. Chem. C, 2013, 117, 7214-7225.

21 J. Canivet, J. Bonnefoy, C. Daniel, A. Legrand, B. Coasne and D. Farrusseng, New J. Chem., 2014, 38, 3102-3111.

22 J. Kim, L. J. Cote, F. Kim, W. Yuan, K. R. Shull and J. Huang, J. Am. Chem. Soc., 2010, 132, 8180-8186.

$23 \mathrm{X} . \mathrm{Hu}, \mathrm{Y} . \mathrm{Yu}, \mathrm{W}$. Hou, J. Zhou and L. Song, Appl. Surf. Sci., 2013, 273, 118-121.

24 J. Cravillon, S. Muenzer, S. Lohmeier, A. Feldhoff, K. Huber and M. Wiebcke, Chem. Mater., 2009, 21, 1410-1412.

25 M. J. C. Ordonez Jr, K. J. Balkus, J. P. Ferraris and I. H. Musselman, J. Membr. Sci., 2010, 361, 28-37.

26 B. N. Bhadra, J. Y. Song, S. Lee, Y. K. Hwang and S. H. Jhung, J. Hazard. Mater., 2018, 344, 1069-1077. 